\section{Multikomponenten-Intervention bremst ungezügelte Opioidverordnung}

\author{
Der Missbrauch verordneter Opioide hat in den USA epidemieartige Ausmaße \\ angenommen. Nun werden alle Register gezogen, um das Verordnungsver- \\ halten der Ärzte leitliniengerechter zu gestalten. Am besten gelingt das wohl \\ mit einer Multikomponenten-Intervention.
}

\begin{abstract}
eitlinien zur Verordnung von LLangzeitopioiden, Schulungsangebote und Implementierung von Programmen zur Opioidrezeptierung alleine können die Opioidmissbrauchswelle in den USA offensichtlich nicht eingedämmen. In einer randomisierten klinischen Studie sollte die Effektivität einer Multikomponentenintervention, bestehend aus einer zusätzlichen Betreuung der Schmerzpatienten durch geschulte medizinische Fachkräfte, elektronischen Entscheidungshilfen und Verordnungsüberwachung sowie regelmäßigen individuellen Performance-Reports mit Rückmeldungsgesprächen, überprüft werden.

Einbezogen wurden 53 Praxen und 985 dort betreute Patienten mit Langzeit-
\end{abstract}

opioidverordnungen aufgrund einer Schmerzindikation. Die Interventionspraxen arbeiteten mit dem Multikomponentenprogramm, in den Kontrollpraxen wurden lediglich elektronische Entscheidungshilfen implementiert. Die primären Studienziele umfassten eine Dokumentation der leitliniengerechten Versorgung über zwölf Monate sowie zwei oder mehr vorzeitige Opioid-„Refills“ (erneutes Einreichen des Rezepts).

Die durchschnittlich verordnete Morphin-Äquivalenzdosis betrug $57,8 \mathrm{mg}$. Nach einem Jahr war die Wahrscheinlichkeit einer leitliniengerechten Opioidverordnung bei den Patienten der Interventionspraxen höher als bei den Patien- ten der Kontrollpraxen (65,9 vs. $37,8 \%$, $\mathrm{p}<0,001$; adjustierte Odds ratio 6,0 , $95 \%$-Konfidenzintervall 3,6-10,2). Bei den frühen Wiederholungsrezepten bestanden keine Unterschiede, allerdings reduzierten die strenger reglementierten Ärzte mit einer größeren Wahrscheinlichkeit die verordneten Opioiddosen um $10 \%$ oder beendeten die Behandlung ganz. In den adjustierten Analysen lag die verordnete Morphinäquivalenzdosis der Rezepte für die Interventionspatienten um 6,8 mg niedriger als bei den Kontrollpatienten $(\mathrm{p}<0,001)$.

Fazit: Mit Unterstützung einer Multikomponentenintervention verordnen niedergelassene Ärzte in den USA Langzeitopioide leitlinienkonformer und reduzieren mit einer größeren Wahrscheinlichkeit die Dosis im Vergleich zu Ärzten ohne diese Kontrollmaßnahmen.

Dr. Barbara Kreutzkamp

Liebschutz JM et al. Improving adherence to long-term opioid therapy guidelines to reduce opioid misuse in primary care. A cluster-randomized clinical trial. JAMA Intern Med 2017;Online 17.Juli

\section{Multidisziplinäre Palliativversorgung: Metaanalyse ergibt positive Effekte}

\section{Der Nutzen einer spezialisierten multidisziplinären Palliativversorgung kann anhand der Lebensqualität gut gemessen werden. Eine Metaanalyse fasste die Lebensqualitätsdaten aus zwölf randomisierten Studien zusammen.}

D ie multidisziplinäre Palliativversorgung ist als effektive Behandlung von terminal Erkrankten allgemein anerkannt. Leitlinienempfehlungen für die umfassende Betreuung beruhen bisher aber auf Expertenmeinungen und systematischen Reviews, sodass quantitative Aussagen etwa zur der Lebensqualität bisher nicht möglich waren. Ein systematisches Review mit angeschlossener Metaanalyse füllt nun diese Lücke.

Aufgenommen wurden zwölf randomisierte kontrollierte, in Krankenhäusern, Hospizen oder im ambulanten Setting durchgeführte Studien mit insgesamt 2.454 erwachsenen Patienten in fortgeschrittenen Krankheitsstadien, die palliativ durch ein spezialisiertes multiprofessionelles Team versorgt worden waren. 1.766 der Patienten hatten Krebs. Primäres Studienziel der Metaanalyse war die Effektstärke bei der Lebensqualität, zusätzlich wurden die gepoolten standardisierten Mittelwertdifferenzen (SMD) der Lebensqualitätsanalyse auf die 29-Item-Global Healthoder die 30-Item-QLQ-Skala übertragen. Die QLQ-C30-Skala umfasst Werte von 0 bis 100 , die minimale Differenz für eine klinische Relevanz beträgt 8,1.

Insgesamt zeigte sich ein kleiner Vorteil zugunsten der Specialist Palliative Care (SMD 0,16, 95\%-Konfidenzintervall [KI] 0,01-0,31, QLQ-C30 Global Health/QoL 4,1, 95\%-KI 0,3-8,2), die Sensitivitätsanalyse zeigte eine SMD von
0,57 (95\%-KI -0,02 - 1,15, Global Health/ QoL 14,6, 95\%-KI -0,5-29,4). Bei Krebspatienten fiel dieser Effekt etwas deutlicher aus, gerade bei den Patienten, die frühzeitig eine multidisziplinäre Palliativpflege erhielten. Die Ergebnisse zur Schmerzreduktion ergaben ebenso wie andere sekundäre Studienendpunkte ein uneinheitliches Bild. Auch erschwerten die heterogene Zusammensetzung bei den Patientenkollektiven und Endpunkten die gepoolte Auswertung.

Fazit: Eine erste Metaanalyse zur spezialisierten multiprofessionellen Palliativversorgung zeigte kleine Effekte bei der Verbesserung der Lebensqualität. Die Versorgung sollte so früh wie möglich und vor allem Patienten mit Versorgungslücken (unmet needs) angeboten werden.

Dr. Barbara Kreutzkamp

Gaertner J et al. Effect of specialist palliative care services on quality of life in adults with advanced incurable illness in hospital, hospice, or community settings: systemic review and metaanalysis. BMJ 2017; 2017;357:j2925 